\title{
A THIRD-ORDER IRREGULAR BOUNDARY VALUE PROBLEM AND THE ASSOCIATED SERIES*
}

BY

\author{
LEWIS E. WARD
}

INTRODUCTION

Published accounts of properties of characteristic functions of third-order, irregular boundary value problems and of expansions in infinite series of such functions have been given by $\mathrm{D}$. Jackson and Hopkins $\dagger$ and by the author. $f$ In these papers were considered only cases which may be regarded as elementary in the sense that the characteristic functions were elementary functions. The present paper deals with the characteristic functions and the associated series of the differential system

$$
d^{3} u / d x^{3}+\left[\rho^{3}+r(x)\right] u=0, \quad u(0)=u^{\prime}(0)=u(\pi)=0 .
$$

We shall suppose throughout that $r(x)$ is a convergent power series in $x^{3}$, and that, when the radius of the circle of convergence of this series is less than $\pi$, $r(x)$ is continuous in the interval $0 \leqq x \leqq \pi$. Many of the methods and results obtained in this paper (but not all of them) can be carried over immediately to the case in which the differential equation is the same as the present one but the boundary conditions are of the type in Part I of the 1927 paper.

The first part of this paper is devoted to a study of the characteristic functions, the second part to necessary conditions for the convergence of a formal series of these functions arising from a given function $f(x)$, and the third part to a statement of conditions sufficient to ensure the convergence to $f(x)$ of the formal series for $f(x)$, and to the proof of such convergence.

\section{PART I}

We first obtain an integral equation equivalent to the differential equation (1) and the boundary conditions $u(0)=u^{\prime}(0)=0$. Let $\omega_{1}=-1, \omega_{2}=e^{\pi i / 3}$, $\omega_{3}=e^{-\pi i / 3}$, and define

$$
\begin{aligned}
& \delta_{1}(t)=e^{\omega_{1} t}+e^{\omega_{2} t}+e^{\omega_{3} t}, \\
& \delta_{2}(t)=e^{\omega_{1} t}-\omega_{3} e^{\omega_{2} t}-\omega_{2} e^{\omega_{3} t}, \\
& \delta_{3}(t)=e^{\omega_{1} t}-\omega_{2} e^{\omega_{3} t}-\omega_{3} e^{\omega_{3} t} . \S
\end{aligned}
$$

* Presented to the Society, October 31, 1931; received by the editors July 28, 1931.

$\dagger$ These Transactions, vol. 20 (1919), p. 245, et seq.

‡ These Transactions, vol. 29 (1927), p. 716, et seq., and vol. 32 (1930), p. 544, et seq. We shall refer to these papers by the year of their publication.

$\$$ See the 1927 paper, p. 720 . 
TheOREM I. A necessary and sufficient condition that $u(x, \rho)$ satisfy $u^{\prime \prime \prime}+\left[\rho^{3}+r(x)\right] u=0, u(0)=u^{\prime}(0)=0$, is that

$$
u(x, \rho)=k \delta_{3}(\rho x)-\frac{1}{3 \rho^{2}} \int_{0}^{x} r(t) \delta_{3}[\rho(x-t)] u(t, \rho) d t,
$$

where $k$ is independent of $x$.

To prove the sufficiency of the condition we differentiate both sides of the integral equation:

$$
\begin{aligned}
u^{\prime}(x, \rho) & =-k \rho \delta_{2}(\rho x)+\frac{1}{3 \rho} \int_{0}^{x} r(t) \delta_{2}[\rho(x-t)] u(t, \rho) d t, \\
u^{\prime \prime}(x, \rho) & =k \rho^{2} \delta_{1}(\rho x)-\frac{1}{3} \int_{0}^{x} r(t) \delta_{1}[\rho(x-t)] u(t, \rho) d t, \\
u^{\prime \prime \prime}(x, \rho) & =-k \rho^{3} \delta_{3}(\rho x)-r(x) u(x, \rho)+\frac{\rho}{3} \int_{0}^{x} r(t) \delta_{3}[\rho(x-t)] u(t, \rho) d t \\
& =-\rho^{3} u(x, \rho)-r(x) u(x, \rho) .
\end{aligned}
$$

To prove the necessity we will show that if $u(x, \rho)$ satisfies the differential equation and if

$$
u(0, \rho)=u^{\prime}(0, \rho)=0, u^{\prime \prime}(0, \rho)=l,
$$

then a value of $k$, independent of $x$, exists such that $u(x, \rho)$ satisfies the integral equation. If we take $k=l /\left(3 \rho^{2}\right)$, then the unique solution of the integral equation is seen to satisfy the differential equation as well as the boundary conditions (2). Hence this unique solution must be identical with the unique solution of the differential equation and equations (2), and this completes the proof.

Without loss of generality we may take $k=1$, and we shall do this. Instead of considering the resulting integral equation we shall take the more general one

$$
u(x, \xi, \rho)=\delta_{3}[\rho(x-\xi)]-\frac{1}{3 \rho^{2}} \int_{\xi}^{x} r(t) \delta_{3}[\rho(x-t)] u(t, \xi, \rho) d t,
$$

as the function defined by the latter enters in Part III. This function reduces to $u(x, \rho)$ upon putting $\xi=0$. Concerning equation (3) we first prove

THEOREM II. If $|\rho|$ is large, and $0 \leqq \xi \leqq x \leqq \pi$, then the solution of (3) is given by

$$
u(x, \xi, \rho)=e^{\omega_{2} \rho(x-\xi)}\left[-\omega_{3}-\omega_{2} e^{\left(\omega_{2}-\omega_{3}\right) \rho(x-\xi)}+\rho^{-2} E(x, \xi, \rho)\right]^{*}
$$

providing $\rho$ remains in the sector $0 \leqq \arg \rho \leqq \pi / 3$.

* Throughout this paper we shall use the notation $E$ to denote any function of the appropriate variables which is bounded when $|\rho|$ is large. 
In (3) let us put

$$
u(x, \xi, \rho)=e^{\omega_{3} \rho(x-\xi)}\left[-\omega_{3}-\omega_{2} e^{\left(\omega_{2}-\omega_{3}\right) \rho(x-\xi)}+z(x, \xi, \rho)\right] .
$$

Then $z(x, \xi, \rho)$ will satisfy the integral equation

$$
\begin{aligned}
z(x, \xi, \rho)=e^{\left(\omega_{1}-\omega_{3}\right) \rho(x-\xi)} & +\frac{1}{3 \rho^{2}} \int_{\xi}^{x} r(t) \delta_{3}[\rho(x-t)] e^{\omega_{3} \rho(t-x)}\left[\omega_{3}+\omega_{2} e^{\left(\omega_{2}-\omega_{3}\right) \rho(t-\xi)}\right] d t \\
& -\frac{1}{3 \rho^{2}} \int_{\xi}^{x} r(t) \delta_{3}[\rho(x-t)] e^{\omega_{3} \rho(t-x)} z(t, \xi, \rho) d t .
\end{aligned}
$$

Since $|z(x, \xi, \rho)|$ is continuous in $x$ and in $\xi$ it will attain its maximum $M$ on the range to which $x$ and $\xi$ are restricted at some point on this range, and at this point we shall have

$$
\begin{aligned}
M \leqq & \left|e^{\left(\omega_{1}-\omega_{8}\right) \rho(x-\xi)}\right| \\
& +\left|\frac{1}{3 \rho^{2}}\right| \int_{\xi}^{x}|r(t)|\left|\delta_{3}[\rho(x-t)] e^{\omega_{3} \rho(t-x)}\right|\left|\omega_{3}+\omega_{2} e^{\left(\omega_{2}-\omega_{3}\right) \rho(t-\xi)}\right| d t \\
& +M\left|\frac{1}{3 \rho^{2}}\right| \int_{\xi}^{x}|r(t)|\left|\delta_{3}[\rho(x-t)] e^{\omega_{3} \rho(t-x)}\right| d t .
\end{aligned}
$$

If we require $\rho$ to be in the sector $0 \leqq \arg \rho \leqq \pi / 3$, we obtain further $M \leqq A /\left|\rho^{2}\right|+M B /\left|\rho^{2}\right|$, where $A$ and $B$ are independent of $x$, $\xi$, and $\rho$. Hence $M \leqq A /\left(\left|\rho^{2}\right|-B\right)$ and $z(x, \xi, \rho)=\rho^{-2} E(x, \xi, \rho)$. This gives the form stated in the theorem.

We shall call the sectors $0 \leqq \arg \rho \leqq \pi / 3$ and $-\pi / 3 \leqq \arg \rho \leqq 0, S_{1}$ and $S_{2}$ respectively. The $u(x, \xi, \rho)$ of Theorem II is, of course, one analytic function of $\rho$ in both sectors. In fact, since $u(x, \xi, \rho)$ is analytic in $\rho$ for every finite $\rho$, and real when $\rho$ is real, the Maclaurin's series for $u(x, \xi, \rho)$ in $\rho$ has real coefficients. Hence $u(x, \xi, \rho)$ takes on in $S_{2}$ values conjugate to those it takes on in $S_{1}$.

The characteristic equation. The characteristic equation is obtained from the third boundary condition (1) and is $u(\pi, \rho)=0$. Its form in $S_{1}$ can be obtained by making use of the asymptotic form of $u(x, \xi, \rho)$ given in Theorem II, and is $-\omega_{3}-\omega_{2} e^{\left(\omega_{2}-\omega_{3}\right) \rho \pi}+\rho^{-2} E(\rho)=0$, where we have discarded an obvious, non-vanishing factor. The function $\phi(\rho)=-\omega_{3}-\omega_{2} e^{\left(\omega_{2}-\omega_{3}\right) \rho \pi}$ has zeros at the points $(1 / 3+2 k) / 3^{1 / 2}$, where $k$ is any integer. If we mark these points, draw small circles all of the same radius about them as centers, and call $S_{1}^{\prime}$ that part of $S_{1}$ which is not interior to these circles, then $|\phi(\rho)|$ has a positive minimum $\delta$ in $S_{1}^{\prime}$. In the part of $S_{1}^{\prime}$ where $\left|\rho^{-2} E(\rho)\right|<\delta / 2$ we have $\left|\phi(\rho)+\rho^{-2} E(\rho)\right|>\delta / 2$, and hence large characteristic numbers in $S_{1}$ can occur only in the circles. 
That there is just one characteristic number in each circle, at least for sufficiently large values of $|\rho|$, is seen as follows. Let $K$ be one such circle, $K_{1}$ the part of $K$ in $S_{1}$ and $K_{2}$ the part in $S_{2}$. As $\rho$ travels around $K_{1}$, $\arg \phi(\rho)$ increases by approximately $\pi$. In fact, $\arg \left[e^{\omega_{3} \rho \pi} \phi(\rho)\right]$ increases by exactly $\pi$, since $e^{\omega_{3} \rho \pi} \phi(\rho)$ is real when $\rho$ is real. Also, during this half circuit, $\left|e^{\omega_{3} \rho \pi} \phi(\rho)\right| \geqq \delta e^{\left(\rho_{k} / 2 \rightarrow r\right) \pi}$, where $r$ is the radius of $K$. At the same time

$$
\left|e^{\omega_{s} \rho \rho^{-2}} E(\rho)\right|<\frac{\delta}{2\left(\rho_{k}-r\right)^{2}} e^{\left(\rho_{k} / 2-r\right) x} .
$$

Since this is a small fraction of $\left|e^{\omega_{3 \rho} \pi} \phi(\rho)\right|$, it follows that $\arg u(\pi, \rho)$ increases by $\pi$ exactly when $\rho$ travels over $K_{1}$. But when $\rho$ travels over $K_{2}$, $u(\pi, \rho)$ takes on values conjugate to those it had on $K_{1}$. Hence arg $u(\pi, \rho)$ increases by $\pi$ more, i.e., when $\rho$ travels around $K, \arg u(\pi, \rho)$ increases by $2 \pi$, and hence $u(\pi, \rho)$ has just one zero inside $K$. This zero is real because of the conjugate values of $u(\pi, \rho)$ in $S_{1}$ and $S_{2}$. We are assured, therefore, of an infinite set of characteristic numbers which can be numbered $\rho_{1}, \rho_{2}, \ldots$, and which, at least for large values of $k$, are real and given asymptotically by $(1 / 3+2 k) / 3^{1 / 2}$.

The characteristic functions. The characteristic functions are now obtained by replacing $\rho$ in the solution of equation (3) by $\rho_{k}$ and $\xi$ by zero, and are denoted by $u_{k}(x)$ so that $u_{k}(x)=u\left(x, 0, \rho_{k}\right)$.

THEOREM III. If $\delta$ is a positive constant, an integer $K$, independent of $x$, can be determined so that

$$
u_{k}(x)=-2 e^{\rho x / 2}\left[\cos \left(\pi / 3+3^{1 / 2} \rho_{k} x / 2\right)+\delta_{k}(x)\right],
$$

where $\left|\delta_{k}(x)\right|<\delta$ if $k>K$, and $x$ is in the interval $0 \leqq x \leqq \pi$.

From Theorem II we have

$$
\begin{aligned}
\boldsymbol{u}_{k}(x) & =-\omega_{2} e^{\omega_{2} \rho_{k} x}-\omega_{3} e^{\omega_{\rho} \rho_{k} x}+e^{\omega_{s} \rho_{k} x} \rho_{k}{ }^{-2} E\left(x, \rho_{k}\right) \\
& =e^{\rho \rho_{k} x / 2}\left[-2 \cos \left(\pi / 3+3^{1 / 2} \rho_{k} x / 2\right)+e^{-i 3^{1 / 2} \rho_{k x} / 2} \rho_{k}{ }^{-2} E\left(x, \rho_{k}\right)\right],
\end{aligned}
$$

and the equation of the present theorem follows immediately from this.

\section{PART II}

ThEOREM IV. If the infinite series

$$
\sum_{k=1}^{\infty} a_{k} u_{k}(x)
$$

converges uniformly in the interval $0 \leqq \alpha \leqq x \leqq x_{0}$, where $\alpha<x_{0}$, and $x_{1}$ is any number less than $x_{0}$, then $\left|a_{k}\right|<h e^{-\rho_{k} x_{1} / 2}$, where $h$ is independent of $k$. 
If $k$ is sufficiently large, we can find a number $x_{k}^{\prime}$ in $\left(x_{1}, x_{0}\right)$ such that $\cos \left(\pi / 3+3^{1 / 2} \rho_{k} x_{k}^{\prime} / 2\right)=1$. Hence $u_{k}\left(x_{k}^{\prime}\right)=-2 e^{\rho_{k} x_{k}^{\prime} / 2}\left[1+\delta_{k}\left(x_{k}^{\prime}\right)\right]$, and $\left|u_{k}\left(x_{k}^{\prime}\right)\right|>e^{\rho k x_{k}^{\prime} / 2}>e^{\rho_{k} x_{1} / 2}$. But $\left|a_{k} u_{k}(x)\right|<h$, where $h$ is independent of $x$ and of $k$. Hence $\left|a_{k}\right|<h e^{-\rho_{k} x_{1} / 2}$. This inequality can be extended to include all values of $k$ by choosing a different $h$ if necessary.

We must now investigate the convergence of (4) for complex values of $x$, and for this purpose we need the asymptotic forms of $u_{k}(x)$ for large $k$ and for $x$ in certain regions to be defined presently. These forms we shall obtain from equation (3), where $x$ is a complex variable, $\xi$ a complex parameter, and $\rho$ a positive constant. The $t$-integration is tc be taken along any simple curve connecting $\xi$ with $x$, and unless the contrary is stated, we shall always take the curve as a single straight line.

We first assure ourselves of the existence of a unique solution of equation (3) analytic in $x$ and also in $\xi$. Define

$$
\begin{aligned}
& K_{1}(x, t)=-r(t) \delta_{3}[\rho(x-t)] /\left(3 \rho^{2}\right), \\
& K_{j}(x, t)=\int_{t}^{x} K_{1}(x, y) K_{j-1}(y, t) d y \quad(j=2,3, \cdots),
\end{aligned}
$$

and consider the infinite series

$$
-K_{1}(x, t)-K_{2}(x, t)-\cdots \text {. }
$$

Each term of this series is analytic in $x$ and also in $t$, at least if we restrict these variables to the circle of convergence of the Maclaurin's series for $r(t)$. We have $\left|K_{1}(x, t)\right|<M$ in this circle. Hence

Assume

$$
\left|K_{2}(x, t)\right|<M^{2} \int_{t}^{x}|d y|=M^{2}|x-t| .
$$

$$
\left|K_{j-1}(x, t)\right|<M^{j-1}|x-t|^{i-2} /(j-2) ! .
$$

This is known to be true if $j=3$. Then

$$
\left|K_{j}(x, t)\right|<\frac{M^{i}}{(j-2) !} \int_{t}^{x}|y-t|^{j-2}|d y|=\frac{M^{i}}{(j-1) !}|x-t|^{j-1} .
$$

Hence $\left|K_{j}(x, t)\right|<L^{i} /(j-1)$ !, where $L$ is a constant. Hence series (5) converges uniformly with respect to both $x$ and $t$, and defines a function $k(x, t)$ analytic in $x$ and also in $t$.

Now consider the function

$$
w(x, \xi, \rho)=\delta_{3}[\rho(x-\xi)]-\int_{\xi}^{x} k(x, t) \delta_{3}[\rho(t-\xi)] d t .
$$


We will show that $w(x, \xi, \rho)$, which is clearly analytic in $x$ and also in $\xi$, satisfies equation (3) by substituting (6) into (3). After cancelling an obvious term we obtain

$$
\begin{aligned}
\int_{\xi}^{x} k(x, t) \delta_{3}[\rho(t-\xi)] d t & =\frac{1}{3 \rho^{2}} \int_{\xi}^{x} r(t) \delta_{3}[\rho(x-t)] \delta_{3}[\rho(t-\xi)] d t \\
& -\frac{1}{3 \rho^{2}} \int_{\xi}^{x} r(t) \delta_{3}[\rho(x-t)] \int_{\xi}^{t} k(t, y) \delta_{3}[\rho(y-\xi)] d y d t .
\end{aligned}
$$

If $x$ and $\xi$ are real, equation (7) can be shown to be true by interchanging the order of integration in the iterated integral and making use of the definition of $k(x, t)$. But both sides of (7) are analytic functions of $x$ and of $\xi$. Hence (7) is true when $x$ and $\xi$ are complex. ${ }^{*}$ This shows that $w(x, \xi, \rho)$ is a solution of (3). That it is the only analytic solution can be shown in the usual way.

We now take $\xi=0$ in (3). Let $\rho$ be large and positive, and $x$ complex. Write $u(x, \rho)=\delta_{3}(\rho x)+e^{\omega_{3} \rho x} z(x, \rho)$. Then $z(x, \rho)$ will satisfy the integral equation

$$
\begin{aligned}
z(x, \rho)= & -\frac{1}{3 \rho^{2}} e^{-\omega_{\rho} \rho x} \int_{0}^{x} r(t) \delta_{3}[\rho(x-t)] \delta_{3}(\rho t) d t \\
& -\frac{1}{3 \rho^{2}} \int_{0}^{x} r(t) \delta_{3}[\rho(x-t)] e^{\omega_{3} \rho(t-x)} z(t, \rho) d t .
\end{aligned}
$$

If we restrict $x$, and consequently $t$ also, to the sector $0 \leqq \arg x \leqq 2 \pi / 3$, we see that all terms arising from the first integral on the right will contain exponentials the real parts of whose exponents are not positive. Also, the terms of the integrand in the second integral, omitting the factor $z(t, \rho)$, will contain similar exponentials. Let us draw a straight line across this sector so as to cut off a finite part $T_{3}$ of the sector such that $r(t)$ has no singularity in or on the boundary of $T_{3}$. We know that $|z(x, \rho)|$ is continuous in $T_{3}$; let it attain its maximum $M$ for $x=x_{2}$ in $T_{3}$. Then $M \leqq \rho^{-2} E_{1}(\rho)+\rho^{-2} M E_{2}(\rho)$, whence $M \leqq E_{1}(\rho) /\left[\rho^{2}-E_{2}(\rho)\right]$, where $E_{1}(\rho)$ and $E_{2}(\rho)$ are bounded. Hence $z(x, \rho)$ $=\rho^{-2} E(x, \rho)$, and we have

Theorem V. If $T_{3}$ is the finite part of the sector $0 \leqq \arg x \leqq 2 \pi / 3$ cut off by a straight line drawn so that $T_{3}$ includes no singularity of $r(t)$, then in $T_{3}$ we have $u(x, \rho)=\delta_{3}(\rho x)+e^{\omega_{3} \rho x} \rho^{-2} E(x, \rho)$, where $E(x, \rho)$ is analytic in $x$ and bounded for $\rho$ large and positive. If $T_{2}$ and $T_{1}$ are regions similarly constructed in the sectors $4 \pi / 3 \leqq \arg x \leqq 2 \pi$ and $2 \pi / 3 \leqq \arg x \leqq 4 \pi / 3$ respectively, then $u(x, \rho)$ $=\delta_{3}(\rho x)+e^{\omega_{2} \rho x} \rho^{-2} E(x, \rho)$ in $T_{2}$, and $u(x, \rho)=\delta_{3}(\rho x)+e^{\omega_{1} \rho x} \rho^{-2} E(x, \rho)$ in $T_{1}$.

* Osgood, Lehrbuch der Funktionentheorie, vol. 2, Part I, p. 24. 
The forms thus given for $T_{2}$ and $T_{1}$ are obtained in a manner similar to that in which the form for $T_{3}$ was obtained.

We may now handle satisfactorily the question of the convergence of (4) for complex values of $x$. Let $T_{3}, T_{2}$, and $T_{1}$ be such that they form an equilateral triangle $T_{x_{2}}$ whose center is at $x=0$ and one vertex of which is at a point $x=x_{2}$ on the positive axis of reals. ${ }^{*}$ Let $x_{2}<x_{1}$. The above forms for $u(x, \rho)$ give us $|u(x, \rho)| \leqq c e^{\rho x_{2} / 2}$, where $c$ is independent of $x$ and of $\rho$, and this is valid throughout $T_{x_{2}}$. Consequently, under the hypotheses of Theorem IV, we have

$$
\left|a_{k} u_{k}(x)\right|<h c e^{\rho k_{k}\left(x_{2}-x_{1}\right) / 2} .
$$

Since this last expression is the general term of a convergent series of positive constants, we may state

THEOREM VI. Under the hypotheses of Theorem IV, the series (4) converges uniformly in the interior and on the boundary of an equilateral triangle $T_{x_{2}}$ centered at $x=0$ and having one vertex $x_{2}$ on the axis of reals between $x=0$ and $x=x_{0}$, providing $r(x)$ has no singularity in or on the boundary of $T_{x_{2}}$.

We make a slight digression from the main course of our argument to prove

THEOREM VII. If $X$ is the upper limit of all possible choices of the $x_{0}$ of Theorem IV, the series (4) cannot converge for any values of $x$ outside of $T_{X}$ except possibly values on the rays $\arg x=0,2 \pi / 3$, or $4 \pi / 3$.

We shall suppose in this connection that $r(x)$ is analytic in such regions as we may desire to use.

Let series (4) converge for $x=x_{2}^{\prime}$, outside of $T_{X}$ and such that $0 \leqq \arg$ $x_{2}^{\prime} \leqq 2 \pi / 3$. Then $\left|a_{k} u_{k}\left(x_{2}^{\prime}\right)\right|<g$, where $g$ is independent of $k$. Also, from the first form in Theorem $\mathrm{V}$,

$$
\begin{aligned}
\left|u_{k}\left(x_{2}^{\prime}\right)\right| & =\left|e^{\omega_{3} \rho k x_{2}^{\prime}}\right| \cdot\left|e^{-\omega_{z} \rho k x_{2}} \delta_{3}\left(\rho_{k} x_{2}^{\prime}\right)+\rho_{k}^{-2} E\left(x_{2}^{\prime}, \rho_{k}\right)\right| \\
& >A^{\prime}\left|e^{\omega_{2} \rho k x_{2}}\right|,
\end{aligned}
$$

where $A^{\prime}$ is independent of $k$. Hence $\left|a_{k}\right|<A\left|e^{-\omega_{3} \rho_{k} x_{2}}\right|$, where $A$ is independent of $k$.

Let $X^{\prime}$ be the point where a line through $x_{2}^{\prime}$ of slope $-3^{-1 / 2}$ cuts the axis of reals, $X^{\prime}>X$, and choose a point $x_{3}$ between $X^{\prime}$ and $X$. Then in the interval $0 \leqq x \leqq x_{3}$ we have $\left|a_{k} u_{k}(x)\right|<B\left|e^{\omega_{3} \rho_{k}\left(x-x_{2}\right)}\right|$, where $B$ is independent of $x$ and of $k$. But $\left|e^{\omega_{3} \rho_{k} x}\right| \leqq e^{\rho_{k} x_{3} / 2}$ and $\left|e^{-\omega_{3} \rho_{k} x^{\prime}}\right|=e^{-\rho_{k} X^{\prime} / 2}$. Hence $\left|a_{k} u_{k}(x)\right|$

\footnotetext{
* We shall consistently mean by the notation $T$ with a literal subscript an equilateral triangle whose center is at $x=0$ and one vertex of which is at the point on the positive axis of reals given by the subscript.
} 
$<B e^{\rho_{k}\left(x_{3}-X^{\prime}\right) / 2}$. Hence series (4) converges uniformly in the interval $0 \leqq x \leqq x_{3}$, and this is a contradiction to our supposition about $X$. Consequently series (4) cannot converge at $x=x_{2}^{\prime}$.

Similar discussions are valid in the sectors $T_{2}$ and $T_{1}$.

Theorem VIII. If $u(x, \xi, \rho)$ satisfies (3), then $u\left(-\omega_{2} x,-\omega_{2} \xi, \rho\right)=$ $-\omega_{3} u(x, \xi, \rho)$.

From the definition of $\delta_{3}(t)$, we have $\delta_{3}\left(-\omega_{2} t\right)=-\omega_{3} \delta_{3}(t)$. Hence, writing $-\omega_{2} x$ and $-\omega_{2} \xi$ in (3) in place of $x$ and $\xi$ respectively,

$$
\begin{aligned}
u\left(-\omega_{2} x,-\omega_{2} \xi, \rho\right)= & -\omega_{3} \delta_{3}[\rho(x-\xi)] \\
& -\frac{1}{3 \rho^{2}} \int_{-\omega_{2} \xi}^{-\omega_{2} x} r(t) \delta_{3}\left[\rho\left(-\omega_{2} x-t\right)\right] u\left(t,-\omega_{2} \xi, \rho\right) d t .
\end{aligned}
$$

Changing the integration variable by the substitution $t=-\omega_{2} t^{\prime}$, and remembering that $r(t)$ is a power series in $t^{3}$, we have

$$
\begin{aligned}
u\left(-\omega_{2} x,-\omega_{2} \xi, \rho\right)= & -\omega_{3} \delta_{3}[\rho(x-\xi)] \\
& -\frac{1}{3 \rho^{2}} \int_{\xi}^{x} r(t) \delta_{3}[\rho(x-t)] u\left(-\omega_{2} t,-\omega_{2} \xi, \rho\right) d t .
\end{aligned}
$$

But this is what (3) would become if we wrote $-\omega_{3} \delta_{3}[\rho(x-\xi)]$ in place of $\delta_{3}[\rho(x-\xi)]$. Hence $u\left(-\omega_{2} x,-\omega_{2} \xi, \rho\right)=-\omega_{3} u(x, \xi, \rho)$.

We are now ready to state the final theorem of this part, which gives conditions on a function that must be satisfied if the function is to be capable of development into a uniformly convergent series of form (4).

THEOREM IX. Under the hypotheses of Theorem IV, series (4) converges to a function analytic at $x=0$ and of the form $x^{2} \phi\left(x^{3}\right)$, where $\phi\left(x^{3}\right)$ is a convergent power series in $x^{3}$.

Let $f(x)$ be the function to which series (4) converges. Since every term of (4) is analytic in $x$ in $T_{x_{2}}$, and the convergence is uniform in this triangle, $f(x)$ must be analytic in this triangle.

From Theorem VIII we see that $u_{k}^{(3 n)}(0)=0$ and $u_{k}^{(1+3 n)}(0)=0$ for all values of $k, n$ being any positive integer or zero. Hence $f^{(3 n)}(0)=f^{(1+3 n)}(0)=0$, where $n$ is any positive integer or zero, and this is equivalent to the special form stated in the theorem.

\section{PART III}

By the formal series for $f(x)$ we mean a series of type (4) in which the $a$ 's are determined by certain orthogonality relations involving the adjoint characteristic functions.* It is known that the sum of the first $n$ terms of the

* For the definition of the adjoint characteristic functions see the fundamental paper by Birkhoff, these Transactions, vol. 9 (1908), p. 373, et seq. 
formal series for $f(x)$ is given by the contour integral

$$
\frac{1}{2 \pi i} \int_{\gamma_{n}} \int_{0}^{\pi} 3 \rho^{2} f(\xi) G(x, \xi, \rho) d \xi d \rho, *
$$

where $G(x, \xi, \rho)$ is the Green's function of the differential system (1), and $\gamma_{n}$ is the arc of a circle centered at $\rho=0$, extending from $\arg \rho=-\pi / 3$ to $\arg \rho=\pi / 3$ and of a radius between $\rho_{n}$ and $\rho_{n+1}$.

A formula for $G(x, \xi, \rho)$ applicable in the present case is given on page 723 of the 1927 paper. The function $g(x, \xi, \rho)$ there defined is given by

$$
g(x, \xi, \rho)= \pm \frac{1}{2} \sum_{j=1}^{3} u_{j}(x) v_{j}(\xi),+ \text { if } x>\xi,- \text { if } x<\xi,
$$

where the $u$ 's are any three independent solutions of the differential equation (1) and $v_{j}(\xi)$ is the cofactor of $u_{j}^{\prime \prime}(\xi)$ in the determinant

$$
W=\left|\begin{array}{lll}
u_{1}^{\prime \prime}(\xi) & u_{2}^{\prime}(\xi) & u_{2}^{\prime \prime}(\xi) \\
u_{1}^{\prime}(\xi) & u_{2}^{\prime}(\xi) & u_{3}^{\prime}(\xi) \\
u_{1}(\xi) & u_{2}(\xi) & u_{3}(\xi)
\end{array}\right|
$$

divided by $W$. The function $\phi(x)=\sum_{j=1}^{3} u_{j}(x) v_{j}(\xi)$ satisfies the following conditions:

(a) $\phi(x)$ is a solution of the differential equation (1) for every $x$ in $0 \leqq x \leqq \pi$,

(b) $\phi(\xi)=\phi^{\prime}(\xi)=0, \phi^{\prime \prime}(\xi)=1$, and these conditions determine $\phi(x)$ uniquely. But the integral equation

(8) $y(x, \xi, \rho)=\frac{1}{3 \rho^{2}} \delta_{3}[\rho(x-\xi)]-\frac{1}{3 \rho^{2}} \int_{\xi}^{x} r(t) \delta_{3}[\rho(x-t)] y(t, \xi, \rho) d t$,

where $\xi$ and $\rho$ are parameters, has a unique solution, and it can be verified immediately that this solution satisfies conditions (a) and (b) above. Hence $\phi(x)=y(x, \xi, \rho)$. Consequently $g(x, \xi, \rho)= \pm y(x, \xi, \rho) / 2,+$ if $x>\xi,-$ if $x<\xi$.

The formula for $G(x, \xi, \rho)$ becomes in the present case

$$
\begin{aligned}
G(x, \xi, \rho)= & -\left|\begin{array}{llll}
u_{1}(x) & u_{2}(x) & u_{3}(x) & g(x, \xi, \rho) \\
u_{1}(0) & u_{2}(0) & u_{3}(0) & g(0, \xi, \rho) \\
u_{1}^{\prime}(0) & u_{2}^{\prime}(0) & u_{3}^{\prime}(0) & g_{x}^{\prime}(0, \xi, \rho) \\
u_{1}(\pi) & u_{2}(\pi) & u_{3}(\pi) & g(\pi, \xi, \rho)
\end{array}\right| \\
& \div\left|\begin{array}{lll}
u_{1}(0) & u_{2}(0) & u_{3}(0) \\
u_{1}^{\prime}(0) & u_{2}^{\prime}(0) & u_{3}^{\prime}(0) \\
u_{1}(\pi) & u_{2}(\pi) & u_{3}(\pi)
\end{array}\right| .
\end{aligned}
$$

* Birkhoff, these Transactions, vol. 9 (1908), p. 379. 
This denominator is recognized as $-W(0) y(\pi, 0, \rho)$. If the first three columns of the determinant in the numerator are multiplied by $v_{1}(\xi) / 2, v_{2}(\xi) / 2$, and $v_{3}(\xi) / 2$ respectively, added to the fourth column, and the resulting determinant expanded according to the elements of the fourth column, the determinant in the numerator is seen to equal

$$
[g(x, \xi, \rho)+y(x, \xi, \rho) / 2] W(0) y(\pi, 0, \rho)-W(0) y(\pi, \xi, \rho) y(x, 0, \rho) .
$$

Hence

$$
G(x, \xi, \rho)=g(x, \xi, \rho)+y(x, \xi, \rho) / 2-y(\pi, \xi, \rho) y(x, 0, \rho) / y(\pi, 0, \rho),
$$

or

$$
G(x, \xi, \rho)= \begin{cases}y(x, \xi, \rho)-y(\pi, \xi, \rho) y(x, 0, \rho) / y(\pi, 0, \rho) & \text { if } x>\xi \\ -y(\pi, \xi, \rho) y(x, 0, \rho) / y(\pi, 0, \rho) & \text { if } x<\xi\end{cases}
$$

Denoting by $I_{n}(x)$ the sum of the first $n$ terms of the formal series for $f(x)$, we now have

$$
I_{n}(x)=\frac{1}{2 \pi i} \int_{\gamma_{n}} 3 \rho^{2}\left[\int_{0}^{x} f(\xi) y(x, \xi, \rho) d \xi-\frac{y(x, 0, \rho)}{y(\pi, 0, \rho)} \int_{0}^{\pi} f(\xi) y(\pi, \xi, \rho) d \xi\right] d \rho .
$$

But a comparison of equations (8) and (3) shows that $3 \rho^{2} y(x, \xi, \rho)=u(x, \xi, \rho)$.

Hence

(9) $I_{n}(x)=\frac{1}{2 \pi i} \int_{\gamma_{n}}\left[\int_{0}^{x} f(\xi) u(x, \xi, \rho) d \xi-\cdot \frac{u(x, 0, \rho)}{u(\pi, 0, \rho)} \int_{0}^{\pi} f(\xi) u(\pi, \xi, \rho) d \xi\right] d \rho$.

Let us write

Then

$$
\sigma(x, s)=\int_{0}^{x} f(\xi) u(s, \xi, \rho) d \xi
$$

and

$$
\int_{0}^{x} f(\xi) u(x, \xi, \rho) d \xi=\sigma(x, x)
$$

$$
\begin{aligned}
\int_{0}^{\pi} f(\xi) u(\pi, \xi, \rho) d \xi & =\int_{0}^{x} f(\xi) u(\pi, \xi, \rho) d \xi+\int_{x}^{\pi} f(\xi) u(\pi, \xi, \rho) d \xi \\
& =\sigma(x, \pi)+\int_{x}^{\pi} f(\xi) u(\pi, \xi, \rho) d \xi .
\end{aligned}
$$

Inserting these in (9), we have

$$
\begin{aligned}
I_{n}(x)= & \frac{1}{2 \pi i} \int_{\gamma_{n}}\left[\sigma(x, x)-\frac{u(x, 0, \rho)}{u(\pi, 0, \rho)} \sigma(x, \pi)\right] d \rho \\
& -\frac{1}{2 \pi i} \int_{\gamma_{n}} \frac{u(x, 0, \rho)}{u(\pi, 0, \rho)} \int_{x}^{\pi} f(\xi) u(\pi, \xi, \rho) d \xi d \rho .
\end{aligned}
$$


We proceed to a study of $\sigma(x, s)$, in which we are interested for $x \leqq s \leqq \pi$.

THEOREM X. The function $\sigma(x, s)$ satisfies the integral equation

$$
\begin{aligned}
\sigma(x, s)=\int_{0}^{x} f(\xi) \delta_{3}[\rho(s-\xi)] d \xi & -\frac{1}{3 \rho^{2}} \int_{0}^{x} r(t) \delta_{3}[\rho(s-t)] \sigma(t, t) d t \\
& -\frac{1}{3 \rho^{2}} \int_{x}^{s} r(t) \delta_{3}[\rho(s-t)] \sigma(x, t) d t .
\end{aligned}
$$

By using (3) we have

$$
\sigma(x, s)=\int_{0}^{x} f(\xi) \delta_{3}[\rho(s-\xi)] d \xi-\frac{1}{3 \rho^{2}} \int_{0}^{x} f(\xi) \int_{\xi}^{s} r(t) \delta_{3}[\rho(s-t)] u(t, \xi, \rho) d t d \xi .
$$

On changing the order of integration in the iterated integral, we obtain

$$
\begin{aligned}
\sigma(x, s)=\int_{0}^{x} f(\xi) \delta_{3}[\rho(s-\xi)] d \xi & -\frac{1}{3 \rho^{2}} \int_{0}^{x} r(t) \delta_{3}[\rho(s-t)] \int_{0}^{t} f(\xi) u(t, \xi, \rho) d \xi d t \\
& -\frac{1}{3 \rho^{2}} \int_{x}^{s} r(t) \delta_{3}[\rho(s-t)] \int_{0}^{x} f(\xi) u(t, \xi, \rho) d \xi d t,
\end{aligned}
$$

and by the definition of $\sigma(x, s)$ this is seen to be equivalent to the integral equation in the statement of the theorem.

If we put $s=x$ in the integral equation of Theorem $\mathrm{X}$, we obtain the integral equation

$$
\sigma(x)=\int_{0}^{x} f(\xi) \delta_{3}[\rho(x-\xi)] d \xi-\frac{1}{3 \rho^{2}} \int_{0}^{x} r(t) \delta_{3}[\rho(x-t)] \sigma(t) d t,
$$

where we have put $\sigma(x, x)=\sigma(x)$. Since (11) is of the same form as (3) for $\xi=0$, we know that (11) has a unique solution analytic in $x$. In fact, we will now prove

THEOREM XI. If $C$ is a circle of radius a centered at $x=0$ and $f(x)$ and $r(x)$ are both analytic inside $C$ and continuous on $C, r(x)$ being a convergent power series in $x^{3}$ and $f(x)$ being $x^{2}$ times such a series, then equation (11) has a solution analytic in $x$ which can be written in the form

where

$$
\sigma(x)=u(x, 0, \rho) \psi_{1}(\rho)+\psi_{2}(x, \rho),
$$

$$
\psi_{2}(x, \rho)=3 f(x) / \rho+E(x, \rho) / \rho^{2} .
$$

We shall replace $\sigma(x)$ in (11) by $u(x, 0, \rho) \psi_{1}(\rho)+\psi_{2}(x, \rho)$ and show that $\psi_{1}(\rho)$ and $\psi_{2}(x, \rho)$ can be defined so that the latter has the stated property. We have 


$$
\begin{aligned}
u(x, 0, \rho) \psi_{1}(\rho)+\psi_{2}(x, \rho)= & \int_{0}^{x} f(\xi) \delta_{3}[\rho(x-\xi)] d \xi \\
& -\frac{\psi_{1}(\rho)}{3 \rho^{2}} \int_{0}^{x} r(t) \delta_{3}[\rho(x-t)] u(t, 0, \rho) d t \\
& -\frac{1}{3 \rho^{2}} \int_{0}^{x} r(t) \delta_{3}[\rho(x-t)] \psi_{2}(t, \rho) d t
\end{aligned}
$$

Making use of (3) with $\xi=0$, and subtracting the term $u(x, 0, \rho) \psi_{1}(\rho)$ from both sides of our equation, leaves

$$
\begin{aligned}
\psi_{2}(x, \rho)= & \int_{0}^{x} f(\xi) \delta_{3}[\rho(x-\xi)] d \xi-\psi_{1}(\rho) \delta_{3}(\rho x) \\
& -\frac{1}{3 \rho^{2}} \int_{0}^{x} r(t) \delta_{3}[\rho(x-t)] \psi_{2}(t, \rho) d t .
\end{aligned}
$$

On integrating by parts twice we have

$$
\int_{0}^{x} f(\xi) \delta_{3}[\rho(x-\xi)] d \xi=3 f(x) / \rho+\rho^{-2} \int_{0}^{x} f^{\prime \prime}(\xi) \delta_{2}[\rho(x-\xi)] d \xi .
$$

We now put into this last integral the value of $\delta_{2}[\rho(x-\xi)]$ and break the integral into the sum of three integrals, and then change the variable of integration in the last two integrals, as we may do because of the analytic character of the integrands. We have

$$
\begin{aligned}
\int_{0}^{x} f^{\prime \prime}(\xi) \delta_{2}[\rho(x-\xi)] d \xi= & e^{\omega_{1} \rho x} \int_{0}^{x} f^{\prime \prime}(\xi) e^{-\omega_{1} \rho \xi} d \xi-\omega_{3} e^{\omega_{2} \rho x} \int_{0}^{x} f^{\prime \prime}(\xi) e^{-\omega_{2} \rho \xi} d \xi \\
& -\omega_{2} e^{\omega_{2} \rho x} \int_{0}^{x} f^{\prime \prime}(\xi) e^{-\omega_{2} \rho \xi} d \xi \\
= & e^{\omega_{1} \rho x} \int_{0}^{x} f^{\prime \prime}(\xi) e^{\rho \xi} d \xi-\omega_{2} e^{\omega_{2} \rho x} \int_{0}^{-\omega_{2} x} f^{\prime \prime}(\xi) e^{\rho \xi} d \xi \\
& -\omega_{3} e^{\omega_{2} \rho x} \int_{0}^{-\omega_{2} x} f^{\prime \prime}(\xi) e^{\rho \xi} d \xi \\
= & \delta_{3}(\rho x) \int_{0}^{y} f^{\prime \prime}(\xi) e^{\rho \xi} d \xi+\mathcal{L} f^{\prime \prime}(\xi) e^{\rho \xi} d \xi,
\end{aligned}
$$

where $y$ is a complex number yet to be defined, and

$$
\mathcal{L} F(t) d t=e^{\omega_{1} \rho x} \int_{y}^{x} F(t) d t-\omega_{2} e^{\omega_{2} \rho x} \int_{y}^{-\omega_{2} x} F(t) d t-\omega_{3} e^{\omega_{2} \rho x} \int_{y}^{-\omega_{2} x} F(t) d t .
$$


It is necessary for $f(x)$ to have the special form mentioned in the theorem in order to obtain this result.

The last integral in (12) is now changed in a manner similar to that in which the first was, except that we do not integrate by parts. This results in

$$
\int_{0}^{x} r(t) \delta_{3}[\rho(x-t)] \psi_{2}(t, \rho) d t=\delta_{3}(\rho x) \int_{0}^{\nu} r(t) e^{\rho} \psi_{2}(t, \rho) d t+\mathcal{L} r(t) e^{\rho} \psi_{2}(t, \rho) d t,
$$

where we have made use of Theorem VIII and of the special form of $r(t)$.

Putting these results back into (12) gives

$$
\begin{aligned}
\psi_{2}(x, \rho)= & 3 f(x) / \rho-\delta_{3}(\rho x)\left[\psi_{1}(\rho)-\frac{1}{\rho^{2}} \int_{0}^{y} f^{\prime \prime}(\xi) e^{\rho \xi} d \xi+\frac{1}{3 \rho^{2}} \int_{0}^{y} r(t) e^{\rho} \psi_{2}(t, \rho) d t\right] \\
& +\frac{1}{\rho^{2}} \mathcal{L} f^{\prime \prime}(\xi) e^{\rho \xi} d \xi-\frac{1}{3 \rho^{2}} \mathcal{L} r(t) e^{\rho t} \psi_{2}(t, \rho) d t .
\end{aligned}
$$

This will certainly be satisfied if

$$
\psi_{2}(x, \rho)=3 f(x) / \rho+\frac{1}{\rho^{2}} \mathcal{L} f^{\prime \prime}(\xi) e^{\rho \xi} d \xi-\frac{1}{3 \rho^{2}} \mathcal{L} r(t) e^{\rho} \psi_{2}(t, \rho) d t
$$

and

$$
\psi_{1}(\rho)=\frac{1}{\rho^{2}} \int_{0}^{\nu} f^{\prime \prime}(\xi) e^{\rho \xi} d \xi-\frac{1}{3 \rho^{2}} \int_{0}^{\nu} r(t) e^{\rho} t \psi_{2}(t, \rho) d t
$$

are both satisfied.

We will now show that equation (13) has a solution with the desired properties. Let

and

$$
f_{1}(x)=3 f(x) / \rho+\rho^{-2} \mathcal{L} f^{\prime \prime}(t) e^{\rho t} d t,
$$

$$
f_{i}(x)=-\frac{1}{3 \rho^{2}} \mathcal{L}^{r}(t) e^{\rho t} f_{j-1}(t) d t \quad(j=2,3, \cdots),
$$

and consider the infinite series

$$
f_{1}(x)+f_{2}(x)+\cdots .
$$

Let $\mu$ be the maximum of $\left|f^{\prime \prime}(t)\right|$ inside and on $C$. Then

$$
\begin{aligned}
& \left|\mathcal{L} f^{\prime \prime}(t) e^{\rho t} d t\right| \leqq \mu\left[\left|e^{\omega_{1} \rho x}\right| \int_{\nu}^{x}\left|e^{\rho t}\right||d t|\right. \\
& \left.\quad+\left|e^{\omega_{\rho} \rho x}\right| \int_{\nu}^{-\omega_{2} x}\left|e^{\rho t}\right||d t|+\left|e^{\omega_{\rho} \rho x}\right| \int_{y}^{-\omega_{2} x}\left|e^{\rho t}\right||d t|\right] .
\end{aligned}
$$

We now take $y=-a e^{-i \arg \rho}$. Then in each of these integrals the integrand 
takes on its largest value at the upper limit. Hence

$$
\left|\mathcal{L}_{f}^{\prime \prime}(t) e^{\rho t} d t\right| \leqq \mu\left[\int_{y}^{x}|d t|+\int_{y}^{-\omega_{2} x}|d t|+\int_{y}^{-\omega_{2} x}|d t|\right] \leqq 6 a \mu .
$$

Hence, $|\rho|$ being large, $\left|f_{1}(x)\right| \leqq M$, and $M$ can be taken so as to be independent of $x$ and of $\rho$.

Now

$$
\begin{aligned}
\left|f_{j}(x)\right| \leqq & \left|\frac{1}{3 \rho^{2}}\right|\left[\left|e^{\omega_{1} \rho x}\right| \int_{y}^{x}\left|e^{\rho t} r(t) f_{j-1}(t)\right||d t|\right. \\
& \left.+\left|e^{\omega_{2} \rho x}\right| \int_{y}^{-\omega_{2} x}\left|e^{\rho t} r(t) f_{j-1}(t)\right||d t|+\left|e^{\omega_{2} \rho x}\right| \int_{y}^{-\omega_{\mathbf{g}} x}\left|e^{\rho t} r(t) f_{j-1}(t)\right||d t|\right] \\
\leqq & \frac{R}{\left|3 \rho^{2}\right|}\left[\int_{y}^{x}\left|f_{j-1}(t)\right||d t|+\int_{y}^{-\omega_{2} x}\left|f_{j-1}(t)\right||d t|+\int_{y}^{-\omega_{2} x}\left|f_{j-1}(t)\right||d t|\right],
\end{aligned}
$$

where $R$ means the maximum of $|r(t)|$ in $C$. Consequently $\left|f_{2}(x)\right| \leqq 2 M R a /\left|\rho^{2}\right|$, and, as can be shown immediately by induction, $\left|f_{i}(x)\right| \leqq 2^{j-1} M R^{j-1} a^{j-1} /$ $\left|\rho^{2 j-2}\right|$. For sufficiently large values of $|\rho|$, therefore, series (15) converges uniformly in $x$ and also in $\rho$.

Let $F(x)$ be the function to which series (15) converges. Then $F(x)$ is analytic in $x$ and continuous in $\rho$. That it is a solution of (13) is seen immediately by putting it into (13) in place of $\psi_{2}(x, \rho)$. We take $\psi_{2}(x, \rho)=F(x)$, thus defining $\psi_{2}(x, \rho)$ uniquely.

We need also the asymptotic form of $\psi_{2}(x, \rho)$. Write $\psi_{2}(x, \rho)=3 f(x) / \rho$ $+v(x, \rho)$. Then $v(x, \rho)$ satisfies the integral equation

$$
v(x, \rho)=\frac{1}{\rho^{2}} \mathcal{L} f^{\prime \prime}(t) e^{\rho t} d t-\frac{1}{3 \rho^{2}} \cdot \mathcal{L} r(t) e^{\rho t}[3 f(t) / \rho+v(t, \rho)] d t .
$$

Let $M$ be the maximum of $|v(t, \rho)|$ in and on $C$. Then $M \leqq\left(\mu_{1}+\mu_{2} M\right) /\left|\rho^{2}\right|$, where $\mu_{1}$ and $\mu_{2}$ are independent of $x$ and of $\rho$. Hence $M \leqq \mu_{1} /\left(\left|\rho^{2}\right|-\mu_{2}\right)$ and $v(x, \rho)=\rho^{-2} E(x, \rho)$. Consequently $\psi_{2}(x, \rho)=3 f(x) / \rho+\rho^{-2} E(x, \rho)$. Insertion of this result into (14) gives $\psi_{1}(\rho)$, and completes the proof.

We must now go back to the integral equation in Theorem $\mathrm{X}$ for $\sigma(x, s)$. Inserting the expression found in Theorem XI for $\sigma(t, t)$, we get

$$
\begin{aligned}
\sigma(x, s)= & \int_{0}^{x} f(\xi) \delta_{3}[\rho(s-\xi)] d \xi-\frac{1}{3 \rho^{2}} \int_{x}^{s} r(t) \delta_{3}[\rho(s-t)] \sigma(x, t) d t \\
& -\frac{1}{3 \rho^{2}} \int_{0}^{x} r(t) \delta_{3}[\rho(s-t)]\left[u(t, 0, \rho) \psi_{1}(\rho)+\psi_{2}(t, \rho)\right] d t .
\end{aligned}
$$

This is an equation for $\sigma(x, s)$ as a function of $s$, and, since $\sigma(t)$ is analytic 
in $t$ so long as $t$ remains in the circle $C$, we are assured of a unique solution analytic in $C$. However, we are interested in a solution for $x \leqq s \leqq \pi$, and if $a<\pi$, then $s$ will be outside of $C$. We wish, then, a solution continuous in $s$ for $x \leqq s \leqq \pi$. We prove

THEOREM XII. If the conditions in Theorem XI are satisfied and if in addition $r(x)$ is continuous for $0 \leqq x \leqq \pi$, then equation (16) has a solution continuous in $s$ for $x \leqq s \leqq \pi$, which can be written in the form

where

$$
\sigma(x, s)=u(s, 0, \rho) \psi_{1}(\rho)+\psi_{3}(x, s, \rho),
$$

$$
\psi_{3}(x, s, \rho)=\left[e^{\omega_{2} \rho(s-x)}+e^{\omega_{s} \rho(s-x)}\right] f(x) / \rho+e^{\omega_{s} \rho(s-x)} \rho^{-2} E(x, s, \rho)
$$

in the sector $0 \leqq \arg \rho \leqq \pi / 3$.

On putting the form for $\sigma(x, s)$ as given in the statement of this theorem into (16) and combining the two terms in $\psi_{1}(\rho)$ on the right hand side, we get

$$
\begin{aligned}
u(s, 0, \rho) \psi_{1}(\rho)+\psi_{3}(x, s, \rho)= & \int_{0}^{x} f(\xi) \delta_{3}[\rho(s-\xi)] d \xi \\
& -\frac{1}{3 \rho^{2}} \int_{0}^{x} r(t) \delta_{3}[\rho(s-t)] \psi_{2}(t, \rho) d t \\
& -\frac{\psi_{1}(\rho)}{3 \rho^{2}} \int_{0}^{s} r(t) \delta_{3}[\rho(s-t)] u(t, 0, \rho) d t \\
& -\frac{1}{3 \rho^{2}} \int_{x}^{s} r(t) \delta_{3}[\rho(s-t)] \psi_{3}(x, t, \rho) d t .
\end{aligned}
$$

If we make use of (3) with $\xi=0$ and $x=s$, we see that we can subtract a common term in $\psi_{1}(\rho)$ from both sides of our present equation, which then becomes

$$
\begin{aligned}
\psi_{3}(x, s, \rho)= & \int_{0}^{x} f(\xi) \delta_{3}[\rho(s-\xi)] d \xi-\frac{1}{3 \rho^{2}} \int_{0}^{x} r(t) \delta_{3}[\rho(s-t)] \psi_{2}(t, \rho) d t \\
& -\delta_{3}(\rho s) \psi_{1}(\rho)-\frac{1}{3 \rho^{2}} \int_{x}^{s} r(t) \delta_{3}[\rho(s-t)] \psi_{3}(x, t, \rho) d t .
\end{aligned}
$$

The first integral on the right hand side is treated in a manner similar to that in which the corresponding integral in the proof of Theorem XI was treated. This gives

$$
\begin{aligned}
\int_{0}^{x} f(\xi) \delta_{3}[\rho(s-\xi)] d \xi=f(x) \delta_{1}[\rho(s-x)] / \rho-f^{\prime}(x) \delta_{2}[\rho(s-x)] / \rho^{2} \\
+\frac{1}{\rho^{2}} \delta_{3}(\rho s) \int_{0}^{\nu} f^{\prime \prime}(\xi) e^{\rho \xi} d \xi+\frac{1}{\rho^{2}} \Re f^{\prime \prime}(t) e^{\rho t} d t,
\end{aligned}
$$


where

$$
\mathcal{R} F(t) d t=e^{\omega_{1} \rho s} \int_{y}^{x} F(t) d t-\omega_{2} e^{\omega_{2} \rho s} \int_{y}^{-\omega_{2} x} F(t) d t-\omega_{3} e^{\omega_{3} \rho s} \int_{y}^{-\omega_{3} x} F(t) d t .
$$

Similarly

$$
\int_{0}^{x} r(t) \delta_{3}[\rho(s-t)] \psi_{2}(t, \rho) d t=\delta_{3}(\rho s) \int_{0}^{y} r(t) e^{\rho t} \psi_{2}(t, \rho) d t+\operatorname{Rr}(t) e^{\rho t} \psi_{2}(t, \rho) d t .
$$

On putting these results into (17) and making use of (14) we find that all terms in $\delta_{3}(\rho s)$ cancel out, leaving

$$
\psi_{3}(x, s, \rho)=f(x) \delta_{1}[\rho(s-x)] / \rho
$$

$$
\begin{aligned}
& -f^{\prime}(x) \delta_{2}[\rho(s-x)] / \rho^{2}+\frac{1}{\rho^{2}} R f^{\prime \prime}(t) e^{\rho t} d t \\
& -\frac{1}{3 \rho^{2}} \operatorname{Rr}(t) e^{\rho t} \psi_{2}(t, \rho) d t-\frac{1}{3 \rho^{2}} \int_{x}^{s} r(t) \delta_{3}[\rho(s-t)] \psi_{3}(x, t, \rho) d t .
\end{aligned}
$$

The cancellation of the terms in $\delta_{3}(\rho s)$ allows us to get from (18) the asymptotic form given for $\psi_{3}(x, s, \rho)$ in the statement of the theorem.

Writing

$$
\psi_{3}(x, s, \rho)=\left[e^{\omega_{2} \rho(s-x)}+e^{\omega_{\beta} \rho(s-x)}\right] f(x) / \rho+e^{\omega_{3} \rho(s-x)} \rho^{-2} v(x, s, \rho),
$$

we have for $v(x, s, \rho)$ the integral equation

$$
\begin{aligned}
v(x, s, \rho)= & \rho e^{\left(\omega_{1}-\omega_{8}\right) \rho(s-x)} f(x)-e^{-\omega_{8} \rho(s-x)} \delta_{2}[\rho(s-x)] f^{\prime}(x) \\
& +e^{-\omega_{8} \rho(s-x)} \mathfrak{R} f^{\prime \prime}(t) e^{\rho t} d t-e^{-\omega_{8} \rho(8-x)} \mathfrak{R}(t) e^{\rho t} \psi_{2}(t, \rho) d t \\
& -\frac{1}{3 \rho} \int_{x}^{s} r(t) \delta_{3}[\rho(s-t)] e^{-\omega_{2} \rho(s-t)}\left[\left\{e^{\left(\omega_{2}-\omega_{8}\right) \rho(t-x)}+1\right\} f(x)\right. \\
& \left.+\frac{v(x, t, \rho)}{\rho}\right] d t .
\end{aligned}
$$

From this equation we see that if $x \leqq s \leqq \pi$, and if $\rho$ is restricted to the sector $0 \leqq \arg \rho \leqq \pi / 3$, then $v(x, s, \rho)$ is bounded when $|\rho|$ is large.

Concerning the $\xi$-integral in equation (10) we prove

THEOREM XIII. If the hypotheses of Theorem XII are satisfied, and in addition $f(x)$ has a continuous second derivative and $0 \leqq x \leqq \pi$, then

$$
\int_{x}^{\pi} f(\xi) u(\pi, \xi, \rho) d \xi=3 f(\pi) / \rho-\left[e^{\omega_{2} \rho(\pi-x)}+e^{\omega_{s} \rho(x-x)}\right] f(x) / \rho+e^{\omega_{2} \rho(\pi-x)} \rho^{-2} E(x, \rho) .
$$

Using equation (3) with $x=\pi$ we obtain 


$$
\begin{aligned}
\int_{x}^{\pi} f(\xi) u(\pi, \xi, \rho) d \xi=\int_{x}^{\pi} f(\xi) \delta_{3}[\rho(\pi-\xi)] d \xi & \\
& -\frac{1}{3 \rho^{2}} \int_{x}^{\pi} f(\xi) \int_{\xi}^{\pi} r(t) \delta_{3}[\rho(\pi-t)] u(t, \xi, \rho) d t d \xi .
\end{aligned}
$$

On integrating by parts twice we find

$$
\begin{aligned}
\int_{x}^{\pi} f(\xi) \delta_{3}[\rho(\pi-\xi)] d \xi= & 3 f(\pi) / \rho-\delta_{1}[\rho(\pi-x)] f(x) / \rho \\
& +\frac{1}{\rho^{2}} \delta_{2}[\rho(\pi-x)] f^{\prime}(x)+\frac{1}{\rho^{2}} \int_{x}^{\pi} f^{\prime \prime}(\xi) \delta_{2}[\rho(\pi-\xi)] d \xi .
\end{aligned}
$$

Hence

$$
\begin{aligned}
& \int_{x}^{\pi} f(\xi) u(\pi, \xi, \rho) d \xi=3 f(\pi) / \rho-\left[e^{\omega_{2} \rho(x-x)}+e^{\omega_{2} \rho(x-x)}\right] f(x) / \rho \\
& +\frac{1}{\rho^{2}} e^{\omega_{\rho} \rho(x-x)}\left[\begin{array}{l}
-\rho f(x) e^{\left(\omega_{1}-\omega_{z}\right) \rho(\pi-x)}+e^{-\omega_{2} \rho(x-x)} \delta_{2}[\rho(\pi-x)] f^{\prime}(x) \\
+e^{-\omega_{2} \rho(\pi-x)} \int_{x}^{\pi} f^{\prime \prime}(\xi) \delta_{2}[\rho(\pi-\xi)] d \xi \\
-\frac{1}{3} e^{-\omega_{2} \rho(\pi-x)} \int_{x}^{\pi} f(\xi) \int_{\xi}^{\pi} r(t) \delta_{3}[\rho(\pi-t)] u(t, \xi, \rho) d t d \xi
\end{array}\right] .
\end{aligned}
$$

Taking account of the asymptotic form of $u(t, \xi, \rho)$ given in Theorem II we see that the quantity in the large brackets is bounded for $|\rho|$ large and $\rho$ in the sector $0 \leqq \arg \rho \leqq \pi / 3$, and thus the theorem is seen to be true.

We are now ready to put our results into equation (10). We shall consider first the $\rho$-integration over the part $\gamma_{n}^{\prime}$ of $\gamma_{n}$ in the sector $S_{1}$. Let $I_{n}^{\prime}(x)$ be the part of $I_{n}(x)$ due to integrating over $\gamma_{n}^{\prime}$. We find that several terms cancel one another. In fact, we arranged our several asymptotic forms so as to be able to see that these very cancellations actually occur. There is left

$$
\begin{aligned}
I_{n}^{\prime}(x)=\frac{1}{2 \pi i} \int_{\gamma_{n}^{\prime}}[3 f(x) / \rho & \left.+\rho^{-2} E(x, \rho)\right] d \rho \\
& -\frac{1}{2 \pi i} \int_{\gamma_{n}^{\prime}} \frac{u(x, 0, \rho)}{u(\pi, 0, \rho)}\left[3 f(\pi) / \rho+e^{\omega_{s} \rho(x-x)} \rho^{-2} E(x, \rho)\right] d \rho .
\end{aligned}
$$

Taking account now of the asymptotic form of $u(x, 0, \rho)$ given in Theorem II, and remembering that in $u(\pi, 0, \rho)=e^{\omega_{3} \rho \pi} E(\rho), E(\rho)$ is not only bounded but has a positive minimum in $S_{1}^{\prime}$, as we saw in the discussion of the characteristic numbers, we have $u(x, 0, \rho) / u(\pi, 0, \rho)=e^{\omega_{3} \rho(x-\pi)} E(x, \rho)$, where $E(x, \rho)$ is bounded for $|\rho|$ large and $\rho$ in $S_{1}^{\prime}$. Hence 


$$
I_{n}^{\prime}(x)=\frac{1}{2 \pi i} \int_{\gamma_{n}^{\prime}}\left[3 f(x) / \rho-e^{-\omega_{z} \rho(\pi-x)} E(x, \rho) / \rho+\rho^{-2} E(x, \rho)\right] d \rho .
$$

From this formula it is clear that $I_{n}^{\prime}(x)=f(x) / 2+\epsilon_{n}(x)$, where $\epsilon_{n}(x) \rightarrow 0$ uniformly as to $x$ when $n \rightarrow \infty$.

As we saw in the discussion following Theorem II, $u(x, \xi, \rho)$ takes on in $S_{2}$ values conjugate to those which it takes on in $S_{1}$, provided $x$ and $\xi$ are real. Hence, from (9), the part of $I_{n}(x)$ arising from the $\rho$-integration in the sector $S_{2}$ equals $I_{n}^{\prime}(x)$. Therefore we have as a culminating theorem

TheOREM XIV. If

(1) $f(x)=x^{2} \phi\left(x^{3}\right)$, where $\phi\left(x^{3}\right)$ is a power series in $x^{3}$ uniformly convergent if $|x| \leqq a \leqq \pi$,

(2) $r(x)$ is a power series in $x^{3}$ uniformly convergent if $|x| \leqq a$,

(3) $f(x)$ has a continuous second derivative in $0 \leqq x \leqq \pi$,

(4) $r(x)$ is continuous in $0 \leqq x \leqq \pi$, then if $a<\pi$, the formal series for $f(x)$ converges uniformly to $f(x)$ in the interval $0 \leqq x \leqq a$; and if $a=\pi$, the formal series for $f(x)$ converges uniformly to $f(x)$ in every interval of the type $0 \leqq x \leqq b<\pi$.

UNIVERSITY OF IOWA, Iowa City, Iowa 